\title{
Acta Haematologica
}

\author{
Systematic Review
}

\section{Systematic Review of Normal Subjects}

\section{Harbouring $B C R-A B L 1$ Fusion Gene}

\author{
Kuan J.W. ${ }^{\text {add }} \cdot$ Su A.T. $^{\text {b }}$ Leong C.F. $^{c} \cdot$ Osato M. $^{\mathrm{d}} \cdot$ Sashida G. $^{\mathrm{d}}$
}

$\underline{\text { Author affiliations }}$

Keywords: $\underline{\text { Chronic myeloid leukaemiaBCR-ABL1Preclinical chronic myeloid }}$

leukaemiaAsymptomatic populationNormal subjects

Acta Haematol 2020;143:96-111

https://doi.org/10.1159/000501146

- ABSTRACT

- GET ARTICLE

- LOGIN / REGISTER

\begin{abstract}
The treatment of chronic myeloid leukaemia (CML) requires quantitative polymerase chain reaction (qPCR) to monitor $B C R-A B L 1$ in International Scale (IS). Some normal subjects were found to harbour $B C R-A B L 1$. We performed a systematic review on normal subjects harbouring BCR-ABL1. A literature search was done on July 16, 2017 using EBSCOhost Research Databases interface and Western Pacific Region Index Medicus. Two authors selected the studies, extracted the data, and evaluated the quality of studies using the modified Appraisal Tool for Cross-Sectional Studies independently. The outcomes were prevalence, level of $B C R$ $A B L 1$ 1s, proportion, and time of progression to CML. The initial search returned 4,770 studies. Eleven studies, all having used convenient sampling, were included, with total of 1,360 subjects.
\end{abstract}


Ten studies used qualitative PCR and one used qPCR (not IS). The mean prevalence of M-BCR was 5.9,15.5, and 15.9\% in cord blood/newborns/infants (CB/NB/I) $(n=170)$, children $(n=90)$, and adults $(n=454)$, respectively, while $\mathrm{m}-\mathrm{BCR}$ was $15,26.9$, and $23.1 \%$ in $\mathrm{CB} / \mathrm{NB} / \mathrm{I}(n=786)$, children $(n=67)$, and adults $(n=208)$, respectively. No study reported the proportion and time of progression to CML. Nine studies were graded as moderate quality, one study as poor quality, and one study as unacceptable. The result of the studies could neither be inferred to the general normal population nor compared. Follow-up data were scarce.

(C) 2019 S. Karger AG, Basel 